certain degree of debility are induced, but these are probably quite as much to be attributed to the disease as to the remedy, and they are always temporary. Of cases in which a cautious course of mercury has proved really injurious to the general health $\mathrm{I}$ have had no experience.

As regards the precise method of administration of mercury, I confess that it does not seem to me a matter of much importance, provided it be done cautiously enough. If by the mouth, the doses must be small-at any rate at first, until the degree of the patient's tolerance has been estimated. I have seen the hypodermic injection plan pursued in Paris and Berlin, but nothing which came under my observation induced me to think favourably of it. The inunction method is safe and very efficient, but it is dirty and inconvenient, and probably nothing can be said in its favour which may not be alleged with greater force of the vapour bath. This latter method, as introduced by $\mathrm{Mr}_{\mathrm{r}}$. Langston Parker and perfected by Mr. Henry Lee, is an admirable plan. Its only drawbacks are the expense and trouble which attend it. At Aix-la-Chapelle, which may, I suppose, in some sense be regarded as a better-class Lock Hospital for the north of Europe, amongst medical men of immense experience in the management of constitutional syphilis, the inunction plan still enjoys undisputed preeminence. From my friends, Dr. Diemer, Dr. Wetzlar, Dr. Brandis, and Dr. Ziemssen, of that place, I heard but one report: all of them believed in mercury as the one cure for syphilis, and inunction as the best method of using it.

But, Mr. President and gentlemen, inasmuch as it was quite as much $\mathrm{my}$ object in undertaking this paper to elicit the opinions of others as to record my own, I am warned that I must hasten to a conclusion. In order that I may not fail in clearness I will, at some risk of being accused of dogmatism, put my conclusions in a categorical shape. They are as follows:-

That mercury is probably a true vital antidote against the syphilitic virus, and that it is capable of bringing about a real cure.

That, in practice, a good many cases are really cured by mercury; the cure being proved by the restoration to good health, and, in some cases, by renewed susceptibility to contagion.

That the probability of cure depends upon the stage of development attained by the disease when the remedy is resorted to, and upon the perseverance with which it is used.

That, in crder to secure the antidotal efficacy of mercury against syphilis, it is desirable to introduce a considerable quantity in to the system, and to protract its use over a very long time.

That ptyalism and other evidences of the physiological action of mercury, so far from being beneficial, are, if possible, to be carefully aroided, since they prevent the sufficiently prolonged use of the remedy.

That in cases in which the patient shows an idiosyncrasy peculiarly susceptible to merenry, the indication is to reduce the dose rather than to omit the drug.

That it is impossible to begin the administration of mercury too soon, and that it should be resorted to without loss of time in all cases in which a chancre shows a tendency to indurate.

That many cases of indurated chancre, treated early by mercury, never show any of the characteristic symptoms of the secondary stage.

That in other cases of mercurial cure of the chancre, in which yet secondary symptoms do occur, they are usually milder than if allowed to develop without specific treatment.

That, when mercury does not wholly abrogate the secondary stage, it exhibits a remarkable power in delaying it.

That delayed outbreaks of secondary syphilis are to be regarded rather as proof that the administration had not been sufficiently persevering than that the remedy was not efficient.

That it is probable that the risk of tertiary symptoms is in ratio with the severity and prolonged duration of the secondary stage.

That there are some grounds for believing that the tertiary symptoms of syphilis are both less frequent and less severe in those who have been efficiently treated by mercury than in others.

That mercury, cautiously given, does not, in a great ma- jority of instances, do any injury to the general health, and that its local inconveniences may usually be prevented.

That the doctrine of the real antidotal character of mercury, in respect to syphilis, ought to lead to much more prolonged administration of it, with the hope of destroying utterly all lingering germs of the malady.

That most collected statistics as to the duration of treatment and freedom from relapse are misleading and worse than useless, because usually the treatment was far too short to be effectual.

That it has not yet been proved that there are any special forms of syphilitic disease in which mercury ought to be avoided, although, as a general rule, it is acknowledged that it must be used with more caution in all forms which are attended by ulceration than in others.

That iodide of potassium possesses little or no efficacy against either the primary or secondary form of syphilis.

That the efficacy of mercury is often most signally proved in cases which have utterly resisted the action of iodide of potassium.

That it does not much matter whether mercury is given by the mouth, by inunction, or by the vapour bath, provided that, whichever method be selected, care be taken to avoid salivation, purging, \&c.

That the doses usually resorted to for internal administration are for the most part too large, and thus often necessitate a premature discontinuance of the remedy.

That if one method of administration does not proceed satisfactorily, another should be tried; and that in no case of difficulty should the vapour bath be forgotten.

\section{THE PROPERTIES, ACTION, AND USES OF CROTON CHLORAL HYDRATE.}

BY J. B URNEY YEO, M.B., M.R.C.P., HON. FELLOW OF RING'S COLLEGE, ASSTSTANT-PHYSICTAN TO KIMG'S COLLEGE AND THE BROMPTON HOSPITALS.

\section{I.-CLINICAL OBSERVATIONS.}

A NEW REMEDT introduced to us by the illustrious discoverer of chloral hydrate naturally commands our attention and interest. Such a remedy is that which Professor Liebreich has named Croton Chloral Hydrate. It is for the purpose of laying before the profession a somewhat systematic investigation, which $I$ have been enabled to make, with regard to the action and uses of this drug that the following paper is written.

It had been stated that remarkable results were to be obtained from the administration of this drug, in one-grain doses, in neuralgia. On the other hand, a German physician who saw me order it in three-grain doses, for a patient in King's College Hospital, derided the smallness of the dose, and assured me that in Germany thirty-grain doses were commonly given; and it seems clear, from Professor Liebreich's own statement, that he has given it in doses of sixty grains.

It was necessary then, in the first place, to endeavour to settle the question of dose. This I hope I have succeeded in doing approximatively.

As I find this substance is very little known amongst medical men and chemists in this country, I may as well occupy a few lines in describing its chief physical properties.

Professor Liebreich tells us that it is formed when chlorine gas acts on aldehyde $\left(\mathrm{C}_{2} \mathrm{H}_{4} \mathrm{O}\right)$; and that its chemical constitution proves it to be the chlorated aldehyde of crotonic acid $\left(\mathrm{C}_{4} \mathrm{H}_{6} \mathrm{O}_{2}\right)$ : so that the formula for croton chloral is $\left(\mathrm{C}_{4} \mathrm{H}_{3} \mathrm{CI}_{3} \mathrm{O}\right)$, that of chloral being $\left(\mathrm{C}_{2} \mathrm{HCl}_{3} \mathrm{O}\right)$.

I'his substance forms small glittering tabular crystals, which tend to cohere together into irregular masses, something after the manner of benzoic acid. It is light (the coherent masses float in water), sparingly soluble in cold, but more so in hot distilled water. It is easily volatilised by heat, but is tolerably permanent at ordinary temperatures. It has a penetrating, mixed camphoraceous and chlorous odour. As the question of its solution is im- 
portant practically, I may mention that $I$ have found the addition of a fourth part of glycerine to warm distilled water greatly increase its solubility. The following formula yields the strongest solution that is permanent; stronger solutions throw down a crystalline deposit on cooling :Croton chloral bydrate, sixty-four grains; glycerine, half an ounce; warm distilled water, an ounce and a half. This gives a solution containing four grains of the drug in each fluid drachm, and having a slightly bitter and camphoraceous taste. I have also given it in pills mixed with an equal part of the confection of roses, and if these are kept in a bottle they form a very good vehicle for its administration.

The method of investigation which seemed to me the most satisfactory was a combination of direct experiment on the lower animals with clinical observations on as many and as various cases as my opportunities afforded me, having regard, of course, to a certain fitness in the cases themselves for the administration and trial of the medicine. I have had no reason to complain of my opportunities; for from a weekly observation of from 500 to 600 out-patients at King's College Hospital, and from 300 to 400 at the Brompton Hospital, together with Dr. Beale's in-patients in King's College Hospital, whom be had for a time entrusted to my care, and my own private patients, it was not difficult to select a considerable number of cases in which the efficacy of croton chloral bydrate could be fairly estimated.

In the physiological laboratory of King's College, by the kindness of Dr. Rutherford, I was enabled to carry out my observations as to the effect of this drug on certain of the lower animals.

The clinical observations being of the chief interest to us as practitioners of medicine, I shall give them precedence in the following statement.

I may divide the cases in which I have administered this drug in to the following classes:-

1. 'To relieve neuralgic pains occurring in the course of the sensory nerves of the face-the branches of the nervus trigeminus.

2. To relieve neuralgic pains occurring in the course of any nerve.

3. To relieve diffused muscular pains.

4. In certain obscure neuroses and spasmodic affections of the nervous system.

5. To allay distressing cough in certain diseases of the respiratory organs.

6. Simply to procure sleep.

Under the first heading I may mention the following :-

CASE 1.-A married lady, with an attack of acute bronchial catarrh, of gouty diathesis, and with a weak heart, complained of severe neuralgic pains extending over the face generally. Had passed a sleepless night from the pain in the face. No carious teeth. To take a grain and a half of croton chloral hydrate every three hours until relieved. An hour and a half after the first dose the pain had disappeared, and no more of the drug was taken, as there was no return of the pain. She slept very soundly through the whole of the following night.

CASE 2. - An anæmic and somewhat hysterical young woman complained of pain coming on every evening in the region of the auriculo-temporal and external frontal nerves. She was ordered two grains of the drug, to be repeated after an hour and a half if not relieved. The first dose did not afford relief ; after the second dose she fell asleep, but awoke with the pain still unmitigated. This process was twice repeated, with the same result. She was urged to double the dose, but refused on the ground of the sleepiness which followed the smaller dose.

CASE 3.-An adult male, in King's College Hospital, recovering from an attack of broncho-pneumonia, complained for two days of severe frontal neuralgia on the left side, spreading upwards over the cranium. To take two grains of croton chloral every three hours. He felt no relief until he had taken eight doses; then the pain was somewhat relieved, and he felt drowsy. After he had taken about twenty doses the pain disappeared entirely, and has not returued.

- CASE 4 was reported to me by Mr. Homan, the housephysician in King's College Hospital. A patient, recovering from a mild attack of typhoid fever, complained of severe pain in the head. Three grains gave almost immediate relief.
CASE 5.-A single young woman, twenty-eight years of age, who had suffered for some months with severe spasmodic cough, probably due to disease of the bronchial glands, complained of troublesome neuralgic pains all over the face. To take two grains, and repeat it after an hour. The pains were relieved by two doses, and the severity of the cough greatly diminished.

CASE 6.-A male out-patient in King's College Hospital complained of severe neuralgic pains arising from decayed teeth. He had had no rest at night for three weeks. He was given five grains, and kept in the hospital for three-quarters of an hour. The pain not being then relieved, another dose of five grains was given. A quarter of an hour after the second dose the pains were quite gone. He was not sleepy. He had two doses given him to take, one on each of the following nights. Five days afterwards he reported that he had had no return of the pain.

CASE 7.-A female out-patient (King's College Hospital), sixty-three years of age, complained of neuralgic pains, extending from the right temporo-maxillary articulation to the tip of the tongue and to the infra-orbital region. To take three grains twice a day. Five days afterwards she reported that she was very much relieved.

CASE 8.-For this case $I$ am indebted to the observations. of Mr. Bartlett, of the Brompton Hospital, who has been kind enough, at my request, to make trial of this remedy in several cases. A young woman, an in-patient in the Brompton Hospital, suffered greatly from neuralgia of the face in connexion with carious teeth. She took two grains, followed after an hour by another grain. The pain was relieved, and she slept.

CASE 9.-A married female out-patient at the Brompton Hospital complained of frontal headache with sleeplessness. Nervons temperament. To take two grains every other night. No effect.

Case 10.-Also reported to me by Mr. Homan. A male in-patient in King's College Hospital with subacute rheumatism, suffered from severe hemicrania. Five grains of croton chloral had no effect. The pain was relieved by a mixture of quinine and bicarbonate of potash.

CASE 11.-A female out-patient at King's College Hospital, aged twenty-six, had suffered from neuralgic pains in the face for three years, with frequent sleepless nights. To take two grains every hour until relieved. Was relieved after four doses, and sleeps much better.

Under my second heading I place the following :-

CASE 1.-A male out-patient at King's College Hospital complained of pains along the upper part of the course of the anterior crural nerve, coming on suddenly in severe paroxysms, and rendering him for the time unable to walk. It lasted two or three hours at a time. He was ordered two grains of croton chloral hydrate night and morning. After taking these doses for three days, he reported the pain as easier at night, but not greatly relieved during the day. As he was suffering much at the time of his second visit to the hospital, he was given five grains and kept in the hospital for an hour; at the end of that time he was much easier, but not at all sleepy. To take five grains every night. Five days afterwards ho reported himself as much better. Sleeps better, and the pain is nearly gone.

CASE 2.-A male out-patient at King's College Hospital, aged forty, a baker, came with severe throbbing pain in the lumbar region. For a fortnight he had been unable to work, and felt severe pain in walking. He was given five grains and kept in the hospital for an hour. The pain was then easier; the throbbing was quite gone; he was not sleepy. To repeat the dose every night. At the end of four days he reported himself as so much better that he was able to return to his work, and walked quite well.

CASE 3.-A male out-patient at King's College Hospital with a very small and superficial painful tumour (neuroma?) in the epigastric region, from which pains darted upwards and backwards to the spine. Had applied belladonna and chloroform liniment. To take two grains of croton chloral and to repeat the dose after an hour if not relieved. Four days afterwards he stated that the pains were relieved by two doses. They returned, but not nearly so severely as before, and were always relieved by the medicine.

CASE 4.-A male out-patient at King's College Hospital, aged fifty-seven. A tedious attack of sciatica had left him with a pain in the left foot, and sleepless at night. "Has not slept for six weeks." To take two grains and a half 
every night. Slept in an hour after the first dose; pains in the foot much better.

CASE 5.-A female out-patient at Brompton Hospital suffering from pleurodynia, hysterical type. To take two grains on alternate nights; no relief.

The following may fall under my third heading :-

CASE 1.-A male out-patient at King's College Hospital had suffered for four months with muscular pains across the hips and in the dorsal region, extending forwards to both sides. They came on after a prolonged gonorrhoa which lasted five months. To take two grains of croton chloral hydrate night and morning. Slept better than usual after the first and third doses; the pain somewhat relieved. To take four grains every night. After four gights he reports that the pain is gone from the hips, but there is still some pain in the dorsal region. To take six grains every night. Four days later be reports that the pain is quite gone from the hips.

CASE 2.-A male in-patient in King's College Hospital with severe rheumatic pains in all the limbs.

Jan. 6th.-2.30 P.Mr.: Five grains of the drug given; no effect.-10 P.M. : Five grains more were given; no effect.

7th.-10 P.M. : Five grains more were given; slept better, but is still restless.

8th.-10 p.M. : Five grains more were given; gave some relief.

CASE 3.-A male out-patient at Brompton Hospital complained of pain in the loins and legs keeping him awake at night. To take two grains of croton chloral on alternate nights. Reports that the pains are much better, and that on the nights he takes the medicine he falls asleep in twenty minutes.

In that indefinite and depressing class of cases which forms my fourth heading I have had little encouragement to proceed in my trials. I may mention, however-

CASE 1.-A male in-patient, at King's College Hospital, of a very hysterical type, was sent in as a case of chorea, but was really suffering from occasional general convulsions, which ceased if the attention of the patient was strongly diverted from himself. Five grains every night produced no effect.

CASE 2.-A lady, beyond middle-age, who had for many years suffered from nocturnal irritability of bladder, aggravated by emotional influences and by cold. Six grains at night produced no effect.

CASE 3.-Reported to me by Mr. Bartleet of the Brompton Hospital. A case of severe dysmenorrhcea was relieved by two doses of one grain each at an interval of an hour.

For the fifth class of cases I believe we have in this drug a valuable adjunct to our other remedies. Out of many in which it has proved of use I would cite the following :-

CASE 1.-A male out-patient at Brompton Hospital, aged thirty-nine, has suffered from asthma all his life. Has now a very distressing cough with great dyspnœa, which makes his nights very restless. To take two grains at bedtime. Reports after a week that the cough and dyspncea are greatly relieved, and that he has slept much better.

CASE 2.-A female out-patient at Brompton Hospital, aged twenty-seven, with advanced phthisical disease in the left lung, suffers much from cough and sleeplessness at night, which the ordinary remedies have not relieved. To take two grains of croton chloral every night. Reports after a week that the cough is much relieved at night and she sleeps well.

CASE 3.-A male out-patient at Brompton Hospital, aged thirty-nine, with emphysema and chronic bronchitis. Cough very distressing at night, which prevents his sleeping, and throws him into a profuse perspiration. To take three grains every night. Reports that the cough and perspirations are both greatly relieved, and that he sleeps much better. I have selected these three, out of a great number, as typical cases.

I have not had opportunities of giving this drug in many eases of simple sleeplessness, and I hesitated to use it for this purpose in large doses until $I$ had seen something of its action on the lower animals.

CASI 1.-Bearing in mind Professor Liebreich's statement that this drug did not affect the action of the heart or respiratory organs, we tried it in a case of severe cardiac disease with chronic broncho-pneumonia, in a young girl in King's College Hospital,. whose symptoms were greatly aggravated by distressing wakefulness. In this case a dose of three grains was quickly followed by sleep.
CASE 2. - A gentleman who occasionally complains of sleeplessness at night, and who is in the habit of obtaining relief from a dose of ten grains of chloral hydrate with ten grains of bromide of potassium, took three grains of croton chloral, and then a grain and a half more at the end of an hour, without any effect. At his own wish he returned to his ordinary dose of chloral hydrate.

I am still continuing systematic observations on the action of this medicine in many cases, but it seemed to me highly desirable that the resalts that have been already obtained should be at once made known, in order that other practical observers might carry on the investigation of the action and uses of what I believe will be found a very valuable therapeutic agent; for in therapeutics, as in other things, it is "in the mouth of many witnesses" that "the truth is estatlished."

I venture to think my clinical observations will, so far as they have gone, support the following conclusions:-

1. In croton chloral hydrate we possess a remedy of remarkable efficacy in some cases of neuralgia of the branches of the nervus trigeminus. I do not remember ever seeing a case similar to Case 6, Class 1, so completely relieved by any medicine, without producing the least drowsiness.

2 . That it also has the power of affording relief in other obstinate forms of neuralgia, as shown in Cases 1, 2, 3, 4, Class 2.

3. That it is of use in certain cases of diffused muscular pain. I would call attention especially to the case of muscular pain after gonorrhœa, Case 1, Class 3. But more evidence is needed to confirm this observation.

4. That it has but little appreciable effect in purely rheumatic cases, as shown in Case 10, Class 1 ; Case 2 , Class 3. I could have added many more cases in support of this statement.

5. That in cases of localised pain and other nerrous symptoms which we find in the class of persons we are in the habit of calling hysterical, this drug is of little or no use. I would call attention, however, to the relief it afforded in a case of dysmenorrhœa (Case 3, Class 4), and suggest that those who are in the habit of seeing many such cases should give this drug a trial.

6. Its efficacy in procuring sleep seems very variable in moderate doses. Two grains will produce sleep in some sensitive females, and ten grains will fail to produce even drowsiness in unsensitive males.

7. Of its value in relieving some forms of irritative and spasmodic cough I have had abundant evidence. If I may trust to the short experience I have hitherto had of it, I should say there is scarcely any remedy that is likely to prove more valuable for the relief of the distressing night cough of chronic phthisis.

Finally, as to the dose. I am satisfied that in dealing with this substance we must give an unusually wide range to the dose, for its effects vary greatly, not only on the human subject, but also, as I shall show hereafter, on the lower animals. The doses $I$ have given varied from one to ten grains. In delicate females I have found very decided effects from doses of two and three grains. In strong males a dose of ten grains is of ten required to produce any appreciable effect. As might have been expected, persons who have been accustomed to the use of anodyne medicine require larger doses than others. The dose must also be proportionate to the sererity and long-continuance of the pain. I would advise that it should be always given in moderate and quickly repeated doses, until the amount of tolerance of the drug in each particular case has been discovered. In severe neuralgias, from two to five grains may be given every hour, or the smaller dose every half-hour, until fitteen grains have been taken. At present $I$ do not think it safe to go beyond this dose. My reason for thinking so will be found in one of the experiments I shall have to narrate, when it will be seen that a dose of twelve grains, injected subcutaneously into a cat, was followed by prolonged unconsciousness, succeeded by attacks of epileptic convulsions, and death after five days. The details of these experiments I propose to reserve for another communication.

Mr. John Wood has kindly permitted me to ascertain the effect of this drug in two cases of cancer of the tongue attended with severe pain, which are now under his care in King's College Hospital, and I shall be able to report the result in my next paper. 